\title{
Abordagem Fisioterapêutica nas Manifestações Neurológicas Decorrentes do Alcoolismo*
}

\author{
Mônica Lavado Moreira** \\ Sissy Veloso Fontes ${ }^{* * *}$ \\ Tatiana Ferraiol Almeida**** \\ Marcia Maiumi Fukujima****
}

\begin{abstract}
RESUMO
O espectro de comprometimento orgânico pelo álcool varia amplamente, sendo seu consumo responsável por distúrbios no sistema nervoso central (ambliopia tabaco-álcool, degeneração cerebelar alcoólica, encefalopatia hepática, encefalopatia de Marchiafava-Bignami, encefalopatia de Wernicke, mielinólise pontina, psicose de Korsakoff) e periférico (neuropatias periféricas), dentre outras. A doença alcoolismo deve ser tratada por equipe multidisciplinar, pois os sinais e os sintomas clínicos são bastante variáveis. Nas instituições de assistência ao alcoólatra visitadas, não foram encontrados profissionais fisioterapeutas, fazendo parte da equipe multidisciplinar. Este estudo conclui que a fisioterapia, pelos seus diversos recursos terapêuticos pode, quando ministrada por fisioterapeuta especializado, prevenir, minimizar e tratar diversas manifestações físicas que esta doença pode acarretar, de modo a colaborar com a reabilitação integral e integrada desses enfermos.
\end{abstract}

\section{UNITERMOS}

Fisioterapia, alcoolismo, instituições especializadas.

\section{NTRODUÇÃO}

Em 1945, Elvin M. Jellinek, psicólogo norte-americano, identificou o alcoolismo como doença, sendo esta reconhecida pela Organização Mundial da Saúde (OMS) somente dois anos mais tarde ${ }^{1}$.

Tanto a identificação como o tratamento de distúrbios relacionados ao uso crônico do álcool são de responsabilidade de vários profissionais da área da saúde, dentre eles médicos, enfermeiros, psicólogos, terapeutas ocupacionais, fonoaudiólogos, assistentes sociais, fisioterapeutas e outros. No entanto, na maioria das vezes, não se suspeita de que muitos desses distúrbios possam estar relacionados diretamente ao uso do álcool, ou são eles diagnosticados quando danos orgânicos extensos e irreversíveis já ocorreram ${ }^{1,2,3}$. Sendo assim, o diagnóstico clínico deve ser realizado o mais precocemente possível, pois além dos danos causados por essa doença, sabemos que, em nosso meio, há elevada incidência de alcoólatras, o que acarreta elevado ônus socioeconômico em países desenvolvidos ou emergentes ${ }^{1,2,3,4,5,6}$. Os profissionais da área da saúde devem estar alertas quanto a esses pacientes, para que sejam tratados na fase inicial da doença, de modo a prevenir danos permanentes e distúrbios psicossociais graves ${ }^{1,7,8}$.

\section{OBJ ETIVO}

Identificar o papel do fisioterapeuta na diagnose e no tratamento preventivo e/ou curativo de pacientes alcoólatras com comprometimento neurológico, em instituições especializadas nesse tipo de atendimento.

\section{MÉTODO}

Pesquisa bibliográfica e de campo. Foram visitadas cinco instituições paulistanas de atendimento ao alcoólatra (Clínica Psiquiátrica Charcot S.A., Programa de Orientação e Atendimento a Dependentes Químicos - PROAD, 


\section{QUADRO 1}

Recursos fisioterapêuticos aplicados para as principais manifestações clínicas do alcoolismo

\begin{tabular}{|c|c|c|}
\hline Doença & Sinais e sintomas & Recursos fisioterapêuticos \\
\hline Degeneração cerebelar alcoólica & $\begin{array}{l}\text { - Déficit de equilíbrio } \\
\text { - Marcha anormal } \\
\text { - Ataxia (mais pronunciada nos } \\
\text { membros inferiores) } \\
\text { - Alteração do tônus } \\
\text { - Hiporreflexia profunda } \\
\text { - Nistagmo } \\
\text { - Disartria }\end{array}$ & $\begin{array}{l}\text { - Cinesioterapia ativa assistida, ativa e resistida } \\
\text { ritmada priorizando a coordenação motora } \\
\text { temporoespacial } \\
\text { - Orientações ao paciente e cuidadores quanto às } \\
\text { atividades de vida diária e de vida prática }\end{array}$ \\
\hline Encefalopatia hepática & $\begin{array}{l}\text { - Alteração do nível de consciência } \\
\text { - Distúrbio do sono } \\
\text { - Apatia } \\
\text { - Marcha atáxica } \\
\text { - Hipertonia } \\
\text { - Paraplegia ou paresia } \\
\text { - Bradicinesia }\end{array}$ & $\begin{array}{l}\text { - Estimulação sensorial } \\
\text { - Massoterapia } \\
\text { - Técnicas de relaxamento } \\
\text { - Cinesioterapia passiva, ativa assistida, ativa ou } \\
\text { resistida ritmada priorizando a coordenação } \\
\text { motora temporoespacial }\end{array}$ \\
\hline Encefalopatia de Wernicke & $\begin{array}{l}\text { - Postura e marcha anormais } \\
\text { - Ataxia } \\
\text { - Confusão mental }\end{array}$ & $\begin{array}{l}\text { - Cinesioterapia ativa assistida, ativa ou resistida } \\
\text { ritmada priorizando a coordenação motora } \\
\text { temporoespacial }\end{array}$ \\
\hline $\begin{array}{l}\text { Encefalopatia de } \\
\text { Marchiafava-Bignami }\end{array}$ & $\begin{array}{l}\text { - Retardo no início da ação motora } \\
\text { - Déficit de memória } \\
\text { - Desorientação temporal e } \\
\text { espacial } \\
\text { - Agitação } \\
\text { - Confusão mental, } \\
\text { alucinação visual }\end{array}$ & $\begin{array}{l}\text { - Cinesioterapia ativa assistida ou ativa com } \\
\text { estratégias de execução cognitivo-motoras, } \\
\text { priorizando pistas mnemônicas (memória } \\
\text { operacional) } \\
\text { - Orientações ao paciente e cuidadores quanto à } \\
\text { ambientação domiciliar e à rotina temporal para } \\
\text { a execução das atividades de vida diária e prática } \\
\text { - Técnicas de relaxamento } \\
\text { - Massoterapia }\end{array}$ \\
\hline Mielinólise pontina & $\begin{array}{l}\text { - Paresia dos músculos da face } \\
\text { - Tetraparesia, podendo evoluir } \\
\text { para tetraplegia flácida e até } \\
\text { para postura descerebrada } \\
\text { - Arreflexia } \\
\text { - Confusão mental }\end{array}$ & $\begin{array}{l}\text { - Exercícios de mímica facial com ou sem auxílio } \\
\text { da crioterapia, tapping ou estimulação elétrica } \\
\text { funcional } \\
\text { - Cinesioterapia passiva, ativa assistida, ativa } \\
\text { ou resistida }\end{array}$ \\
\hline Psicose de Korsakoff & $\begin{array}{l}\text { - Desordem das funções mnêmônicas } \\
\text { - Ataxia } \\
\text { - Nistagmo }\end{array}$ & $\begin{array}{l}\text { - Cinesioterapia ativa assistida, ativa ou resistida } \\
\text { com estratégias de execução cognitivo-motoras, } \\
\text { priorizando pistas mnemônicas (memória } \\
\text { operacional) e ritmada, priorizando a coordenação } \\
\text { motora temporoespacial }\end{array}$ \\
\hline Neuropatia periférica por álcool & $\begin{array}{l}\text { - Disestesias } \\
\text { - Paresia } \\
\text { - Alteração do reflexo tendíneo } \\
\text { - Hipo ou atrofia muscular } \\
\text { - Edema }\end{array}$ & $\begin{array}{l}\text { - Cinesioterapia ativa assistida, ativa ou resistida } \\
\text { - Estimulação sensitiva por variáveis térmicas } \\
\text { (frio e calor), de peso e de textura; tapping; } \\
\text { escovação; percussão; placing } \\
\text { - Drenagem linfática (técnica de massoterapia, } \\
\text { enfaixamentos, banhos de contraste de } \\
\text { temperaturas e orientações quanto a } \\
\text { posicionamentos de drenagem linfática) }\end{array}$ \\
\hline
\end{tabular}


Alcoólicos Anônimos - AA, Al-Anon e Alateen) e entrevistados os seguintes profissionais desses serviços: três médicos (dois psiquiatras e um clínico geral), um psicó$\operatorname{logo}$, dois terapeutas ocupacionais e um assistente social.

\section{RESULTADOS}

As instituições foram classificadas segundo o tipo de assistência prestada.

A clínica psiquiátrica Charcot S.A. e a PROAD possuem equipe multidisciplinar que ministram tratamento especializado, inclusive com internação (leitos particulares e conveniados com o Serviço Único de Saúde - SUS); o AA, o Al-Anon e o Alateen possuem grupos de auto-ajuda coordenados por profissionais especializados.

O profissional fisioterapeuta não foi encontrado compondo as equipes multiprofissionais, em nenhuma das instituições visitadas.

Os problemas psicossociais que o paciente e seus familiares enfrentam devido a essa doença são extremamente graves.

Os recursos terapêuticos utilizados pela fisioterapia sugeridos, nesta pesquisa, são apresentados no quadro 1, segundo os sinais e sintomas apresentados nas principais manifestações neurológicas do alcoólatra.

\section{DISCUSSÃO}

Observamos que a atuação do fisioterapeuta junto a essas equipes é de grande relevância, devido aos inúmeros déficits neurossensomotores que os pacientes alcoólatras podem apresentar.

Os problemas de ordem psicossocial fazem com que haja a necessidade de profissionais variados, atuando conjuntamente, não apenas em suas respectivas especialidades, como também no processo de inclusão social desses pacientes.

Os recursos terapêuticos da fisioterapia indicados no quadro 1 foram selecionados na literatura e sugeridos visando à prevenção, à minimização ou ao tratamento dos déficits que podem ocorrer em quaisquer das doenças listadas causadas pelo alcoolismo. Tais recursos podem ser utilizados de forma isolada ou combinada e devem ser ministrados segundo a necessidade individual de cada caso. Quem prescreve e ministra tais recursos é o profissional fisioterapeuta, especializado nesse tipo de terapêutica e atendimento.

\section{CONCLUSÃO}

Concluímos que o profissional fisioterapeuta não vem sendo utilizado em nenhuma das instituições visitadas e este deve fazer parte da equipe multidisciplinar que presta assistência tanto a esses doentes como aos seus familiares. O fisioterapeuta, além de tratar seqüelas neuromotoras, é mediador do processo de inclusão social de pacientes, o que o torna um profissional de grande valia nessas instituições de assistência aos alcoólatras.

\section{SUMMARY \\ Physiotherapy management in neurologic manifestations of alcoholism There are many clinical manifestations of chronic alcohol consumption, specially in nervous system: tobacco alcohol ambliopy, cerebellar degeneration, hepatic encephalopathy, Wernicke encephalopathy, peripheral neuropathy and others. We visited five Brazilian institutions that care for chronic alcoholists and found that there is no physical therapist in these institutions. We identify the function of these professionals in specific treatments and found that they are needed to compound the therapeutic group, so they could help the patient to achieve a global rehabilitation.}

\section{KEYWORDS}

Physiotherapy, alcoholism, alcoholism institutions.

\section{Referências}

1. Fortes JRA, Cardo WN. In: Alcoolismo: diagnóstico e tratamento. Ed. Sarvier, São Paulo, 1991.

2. Ramos SP, Bertolote JP. In: Alcoolismo hoje. Ed. Artes Médicas Sul Ltda., Porto Alegre, 1997.

3. Gitlow S, Peyser H. In: Alcoolismo: um guia prático de tratamento. Ed. Artes Médicas Sul Ltda., Porto Alegre, 1991.

4. Hirata ES, Almeida OP, Funari RR, Klein EL. Alcoholism in a geriatric outpatients clinic of São Paulo-Brasil. Int Psychogeriatric, 9(1):95-103, 1997.

5. Chaieb JÁ, Castellarian C. Smoking associated with alcoholism: introduction to the major human dependencies. Rev Saúde Pública, 32(3):246-54, 1998.

6. Reid MC, Fiellin DA, Oconnor PG. Hazardous and harmful alcohol consumption in primary care. Arch Intern Med, 159(15):1681-9, 1999.

7. Foster JH, Powel JE, Marshall EJ, Peters TJ. Quality of life in alcohol-dependent subjects: a review. Qual Life Res, 8(3):255-61, 1999

8. Marino A, Costa R. Alcoholism - Aspects of its pharmacology, clinical picture and therapy. Recenti Prog Med, 84(10):70921, 1993.

9. Benjamin K. Doenças neurológicas e do comportamento. In: Wyngaardeen JB, Smith LH. Cecil - Tratado de medicina interna. Ed. Interamericana, Rio de Janeiro, 1984, p. 20482054.

10. Sherlock S. In: Doenças do fígado e do sistema biliar. Ed Guanabara Koogan, Rio de Janeiro, 1990.

11. Prante AJ, Castellano FFJ. In: Álcool e aparelho locomotor. Rev Bras Reum, 31:79-85,1991.

12. Ehrenreich $\mathrm{H}$, Mangholz A, Scmitt M, Lieder P, Völkel W, Rüther E, Poser W. OLITA: an alternative in the treatment of therapy-resistant chronic alcoholics - First evaluation of a new approach. Eur Arch Psychiatry Clin Neurosci, 241(1):514, 1997 
13. Niederhauser $\mathrm{HU}$, Schoch HK. The alcoholic patient in rehabilitation. Ther Umsch, 47(5):405-11, 1990.

14. Edwards S. In: Neurological physiotherapy - A problemsolving approach. Churchill Livingstone, New York, 1996.

15. Nieda KV, Michlovitz SL. Cryotherapy. In: Michlovitz SL. Thermal agents in rehabilitation. F.A. Davis Company, Philadelphia, 1996.

\section{Endereço para correspondência:}

Marcia Maiumi Fukujima

Unifesp-EPM - Disciplina de Neurologia

Rua Botucatu, 740 - Vila Clementino

04023-900 - São Paulo, SP

E-mail:maiumi@sti.com.br/sissyfontes@sti.com.br 